\title{
Bembix rostrata (L.) (Hymenoptera, Crabronidae) de retour en Wallonie (Belgique)
}

\author{
Par Yvan BARBIER ${ }^{*}$
}

\section{Observations en Wallonie}

Le Bembix rostré (Bembix rostrata) (Figure 1) est une espèce emblématique. C'est un des plus gros Crabronidae vivant dans nos régions. Comme les autres espèces de la famille, elle est solitaire. La femelle creuse des nids dans des endroits sablonneux qu'elle approvisionne en proies pour nourrir les larves. Contrairement à la plupart des espèces de la famille, elle approvisionne les larves régulièrement tout au long de leur vie larvaire. Les proies sont composées de diverses espèces de diptères, principalement des Tabanidae et des Syrphidae. On observe souvent les nids de plusieurs femelles au même endroit mais cellesci ne coopèrent jamais.

L'espèce est largement répandue en Europe et en Asie (Bitsch et al., 1997; Barbier, 2004). On la rencontre de l'Afrique du nord aux pays scandinaves. En Asie, sa distribution s'étend jusqu'en Mongolie. Bien qu'ayant une large distribution, Bembix rostrata n'est pas une espèce ubiquiste. Elle affectionne les milieux sablonneux où elle peut être localement abondante.

En Belgique, B. rostrata est bien connu du nord du pays: Campine et littoral (Leclerq, 1978). C'est dans ces régions qu'elle trouve le plus grand nombre d'habitats favorables. La carte de répartition de Leclercq (1978) montre que l'espèce n'est cependant pas très répandue et que plusieurs carrés UTM ne sont représentés que par des anciennes données, antérieures à 1950. En 1980, l'espèce ne figurait pas dans la "liste rouge" établie par Leclercq et al. (1980). En Wallonie, elle est légalement protégée depuis le décret du 6 décembre 2001.

En Allemagne, l'espèce figure sur la liste des espèces menacées (Haeseler \& Schmidt, 1984; Jacobs, 2000); elle est considérée comme "menacée de disparition" en Mecklembourg-Poméranie occidentale et "en danger" dans le Brandebourg (Jacobs, 2000); "très en danger" en Rhénanie-Palatinat (Schmid-Egger et al., 1995) ainsi qu'en Rhénanie-du-Nord-Westphalie (Cölln et al., 2004) et en Bavière (Wickl et al., 2004).

En Wallonie, la carte de Leclercq (1978) montre seulement deux points. Le premier à Anseremme (prov. Namur) en 1945 et le second à Torgny (prov. Luxembourg) après 1950 (Banque de données fauniques de Gembloux et Mons).

\footnotetext{
* Faculté universitaire des Sciences agronomiques de Gembloux. Unité de gestion des ressources forestières et des milieux naturels. Passage des déportés, 2. B-5030 Gembloux. E-mail: yvan.barbier@gmail.com
}

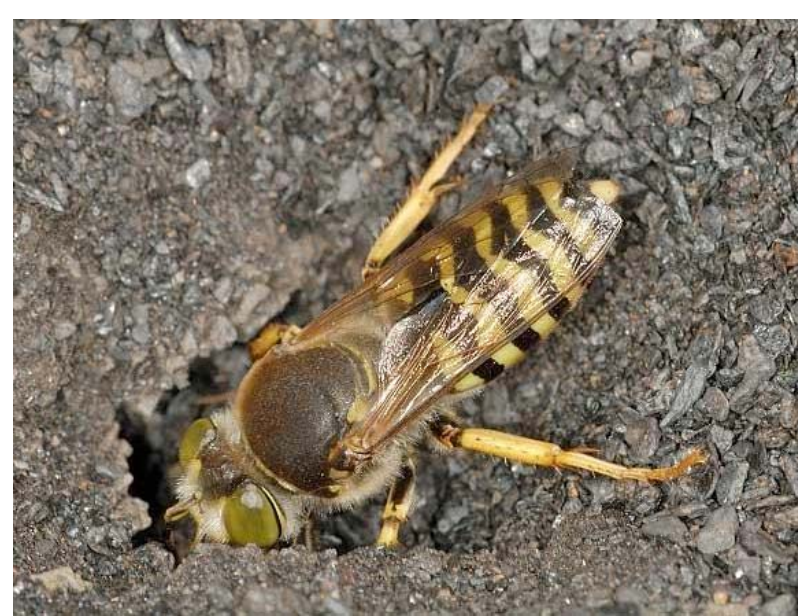

Figure 1. Femelle de Bembix rostrata à l'entrée de son nid. Terril d'Hensies (B), 23.VI.2005 (Photo Y. Barbier)

A part ces deux données anciennes, B. rostrata n'a été trouvé qu'à une seule reprise en Wallonie. C'était à Chantemelle (prov. Luxembourg) en 1990 (J.-P. Jacob, comm. pers.).

En 2005 et en 2006, nous avons eu la surprise de rencontrer cette espèce à deux reprises en Région wallonne. La première fois au Terril d'Hensies (Hainaut) et la seconde fois au Camp militaire de Lagland (Luxembourg).

Au Terril d'Hensies, c'est une dizaine de femelles occupées à approvisionner des nids avec des Tabanidae qui ont été observées en juin 2005. Le Terril d'Hensies est un ancien site industriel situé à l'ouest de la province de Hainaut, dans la commune d'Hensies (UTM: 31UER4889). Il fait partie du vaste complexe des Marais d'Harchies-Hensies-Pommeroeul. Ce terril est atypique: il est plat et sa plus grande partie est constituée de schistes fins et noirs dont la granulométrie est relativement proche de celle d'un sable (Barbier et al., 1990). Ces schistes résultent du traitement du charbon qui s'est fait sur le site jusqu'en 1976, année de la fin de l'exploitation charbonnière. La granulométrie du substrat convient parfaitement aux espèces psammophiles qui peuplent cet habitat artificiel. La diversité et, surtout, l'originalité de l'entomofaune du Terril d'Hensies est en effet en cours d'étude (Barbier, in prep.).

Au camp militaire de Lagland, c'est en juin 2006 que nous avons pu observer 3 femelles volant et se posant sur le sable. Aucun nid n'a pu être observé. Les 3 individus ont tous été vus au lieu dit "la sablière" (UTM 31UFR9703). 


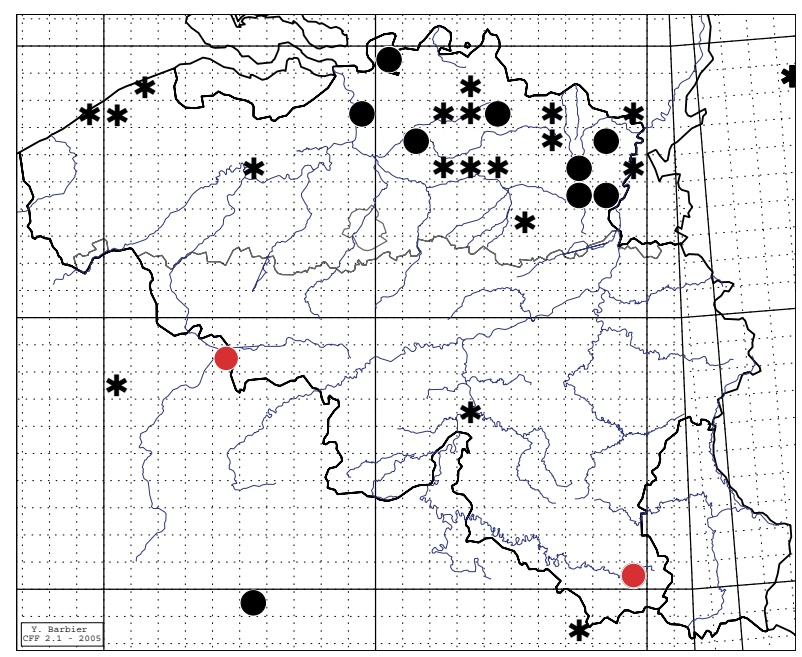

Bembix rostrata

\section{* Avant 1950}

* Avant 1950

Figure 2. Distribution des observations de Bembix rostrata en Belgique. Les sites de Lagland (Luxembourg) et d'Hensies (Hainaut) sont indiqués en rouge sur la carte (lllustration Y. Barbier)

Le camp militaire de Lagland est connu pour ses vastes espaces sablonneux. Ces espaces sont plus ou moins conquis par la végétation. $B$. rostrata a été observé sur le sable nu, à proximité d'une bande partiellement couverte de graminées et de callune (Calluna vulgaris).

Vraisemblablement, B. rostrata est présent dans le sud de la Wallonie depuis longtemps. Ses effectifs ont probablement toujours été faibles et ses populations localisées (Figure 2). Les habitats propices à son installation sont en effet assez nombreux et l'atlas de Leclercq (1978) atteste sa présence ancienne. Par contre, sa présence au Terril d'Hensies est une surprise. Ce site a fait l'objet de nombreuses prospections de la part de l'auteur depuis 1988 et, depuis cette première année, l'espèce n'avait jamais été observée. On peut donc raisonnablement penser que la colonisation du Terril d'Hensies par B. rostrata est très récente.

\section{Références bibliographiques}

Barbier Y, 2004. Fauna Europaea: Sphecidae. Dans Fauna Europaea: Sphecidae (eds Noyes J \& Nieukerken EJ van), Fauna Europaea version 1.1., http://www.faunaeur.org

Barbier Y, Rasmont P \& Wahis R, 1990. Aperçu de la faune des Hyménoptères Vespiformes de deux terrils du Hainaut occidental. Notes fauniques de Gembloux 21: 23-38.

Bitsch J, Barbier Y, Gayubo SF, Schmidt K \& Ohl M, 1997. Faune de France. Hyménoptères Sphecidae d'Europe Occidentale, Volume II. Fédération Française des Sociétés des Sciences Naturelles, Paris, 429 pp, 76 planches, 154 cartes.

Cölln K, Esser J, Fuhrmann M, Jacobi B, Jakubzik, Quest M, Sonnenburg $\mathbf{H}$, Steven M, Tumbrinck K, Wolf $\mathbf{H} \&$ Woydak HG, 2004. Stechimmen in Nordrhein-Westfalen. Ökologie, Gefährdung, Schutz. - Landesanstalt für Ökologie, Bodenordnung und Forsten Nordrhein-Westfalen, Schriftenreihe, Band 20, 327 p.

Haeseler V \& Schmidt K, 1984. Rote Liste der Grabwespen (Sphecoidea). Rote Liste des gefährdeten Tiere und Pflanzen in der Bundesrepublik Deutschland, Greven 4. Auflage, pp. 47-49.

Jacobs H-J, 2000. Rote Liste der gefährdeten Grabwespen Mecklenburg-Vorpommerns (Hymenoptera Aculeata: Sphecidae). 1. Fassung. Das Umweltministerium des Landes Mecklenburg-Vorpommerns. 20pp.

LeclercqJ, 1978. Hymenoptera Sphecidae (cartes 1165-1200). Dans Atlas provisoire des insectes de Belgique (et des régions limitrophes) (eds Leclercq J, Gaspar C \& Verstraeten C). Faculté des Sciences Agronomiques de Gembloux, Zoologie générale et Faunistique, cartes 1001-1200.

Leclercq J, Gaspar G, Marchal J-L, Verstraeten G \& Wonville G, 1980. Analyse des 1600 premières cartes de l'Atlas Provisoire des Insectes de Belgique, et première liste rouge d'insectes menacés dans la faune belge. Notes Fauniques de Gembloux 4: 104 pp.

Schmid-Egger C, Risch S \& Niehuis O, 1995. Fauna und Flora Rheinland-Pfalz. Zeitschrift für Naturschutz. Beiheft 16. Die Wildbienen und Wespen in Rheinland-Pfalz (Hymenoptera, Aculeata). Verbreitung, Ökologie und Gefährdungssituation. Gesellshaft für Naturschutz und Ornithologie, Rheinland-Pfalz e.V. (GNOR), Landau, 296 pp.

Wickl K-H, Voith J, Mandery K, Weber K \& Kraus M, 2004. Rote Liste gefährdeter Grabwespen (Hymenoptera: Sphecidae) Bayerns. Dans Rote Liste gefährdeter Tiere Bayerns. - Schriftenreihe Bayerisches Landesamt fir Umweltschutz, Augsburg 166: 193-197.

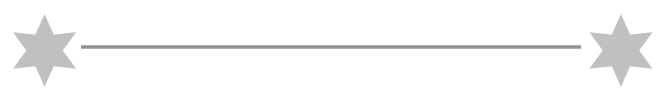

